Doris Cecilia Farfán Del Carpio

\title{
DEGRADAÇÃO FÍSICO-QUIMICA DO PVC CAUSADA POR DERIVADOS DE PETROLEO
}

\section{Dissertação de Mestrado}

Dissertação apresentada como requisito parcial para obtenção do grau de Mestre pelo Programa de PósGraduação em Engenharia de Materiais e de Processos Químicos e Metalúrgicos da PUC-Rio.

Orientador: Prof. José Roberto Moraes d'Almeida 
Doris Cecilia Farfán Del Carpio

\section{DEGRADAÇÃO FíSICO - QUÍMICA DO PVC CAUSADA POR DERIVADOS DE PETRÓLEO}

Dissertação apresentada como requisito parcial para obtenção do grau de Mestre pelo Programa de PósGraduação em Engenharia Materiais e de Processos Químicos e Metalúrgicos da PUC-Rio. Aprovada pela Comissão Examinadora abaixo assinada.

Prof. José Roberto Moraes d'Almeida

Orientador

Departamento de Ciência dos Materiais e Metalurgia - PUC-Rio

Prof. Roberto Ribeiro de Avillez

Departamento de Ciência dos Materiais e Metalurgia - PUC-Rio

Profa. Veronica Calado

Escola de Quimica - UFRJ

Prof. Marcos Henrique de Pinho Mauricio

Departamento de Ciência dos Materiais e Metalurgia - PUC-Rio

(suplente)

Prof. José Eugênio Leal Coordenador Setorial de Pos -graduação do Centro Técnico Científico da PUC-Rio

Rio de Janeiro, 16 de abril de 2009 
Todos os direitos reservados. É proibida a reprodução total ou parcial do trabalho sem autorização da universidade, do autor e do orientador.

\section{Doris Cecilia Farfán Del Carpio}

Engenheira Química (Facultade Engenharia de Processos da Universidade Nacional de San Agustín Arequipa - Perú, 2002)

Ficha Catalográfica

\section{Farfán del Carpio, Doris Cecilia}

Degradação físico-quimica do PVC causada por derivados de petróleo / Doris Cecilia Farfán Del Carpio ; orientador: José Roberto Moraes d'Almeida. -2009 .

112 f. : il. (col.) ; $30 \mathrm{~cm}$

Dissertação (Mestrado em Engenharia de Materiais)-Pontifícia Universidade Católica do Rio de Janeiro, Rio de Janeiro, 2009.

Inclui bibliografia

1. Engenharia de materiais - Teses. 2. PVC. 3. Termogravimetria. 4. Calorimetria diferencial de varredura. 5. Análise FTIR. 6. DRX. 7. Envelhecimento. I. Almeida, José Roberto Moraes d'. II. Pontifícia Universidade Católica do Rio de Janeiro. Departamento de Engenharia de Materiais. III. Título. 


\section{Agradecimentos}

A José Roberto Moraes d'Almeida, meu professor, pela paciência e apoio durante estes dois anos e a sua esposa Ana Fampa.

Ao CNPq, e à PUC-Rio, através da Vice Reitoria Acadêmica (VRAc), pelos auxílios concedidos, sem os quais este trabalho não poderia ter sido realizado.

À Professora Verônica Calado e o pessoal do LADEQ (Departamento de Engenharia Química) da UFRJ.

Aos professores e funcionários do DEMa, por todo apoio didático e administrativo.

Ao Pe. Djalma Rodrigues de Andrade , Reitor da Igreja Sagrado Coração da PUCRio por ser presença em inumeráveis momentos. Ao pessoal da Pastoral, por terem as portas sempre abertas.

À toda minha família, especialmente ao Chatin, meu irmãozinho, aos meus amigos pelo apoio e com gratitude eterna a Walter.

A DEUS, pela minha saúde e por sua infinita generosidade.

A todos, obrigada! Este mestrado não teria sido possível sem a ajuda de vocês. 


\section{Resumo}

Farfán Del Carpio, D. C.; Moraes d'Almeida J. R. Degradação físicoquímica de PVC causada por derivados de petróleo. Rio de Janeiro, 2009. 112p. Dissertação de Mestrado - Departamento Engenharia de Materiais e de Processos Químicos e Metalúrgicos, Pontifícia Universidade Católica do Rio de Janeiro.

O PVC é o único polímero de grande consumo que não é $100 \%$ originário do petróleo, pois contêm, em peso, $57 \%$ de cloro (derivado do cloreto de sódio sal de cozinha) e apenas os restantes $43 \%$ de eteno são derivados do petróleo. Como as matrizes ricas em cloro são privilegiadas para aplicações em meios ambientes ricos em bactérias, tais como dutos enterrados ou imersos em água do mar, o PVC já vem sendo usado em diversas aplicações na área de transporte de fluidos, notadamente água, mas pode ser considerado também uma alternativa para o transporte de outros fluidos. Assim sendo este trabalho estuda o efeito físico - químico do envelhecimento do poli (cloreto de vinila) - PVC, utilizando amostras em pó, expostas a diversos fluidos (água, etanol e diesel), por diversos períodos de tempo. A primeira parte do trabalho consistiu na caracterização do material como recebido e a segunda na caracterização do material após exposição aos fluidos. As técnicas de caracterização utilizadas foram termogravimetria (TGA), termogravimetria diferencial (DTGA), calorimetria diferencial de varredura (DSC), métodos espectroscópicos (FTIR-ATR) e de Difração de Raios $\mathrm{X}$, que permitiram avaliar a interação físico-química dos diversos fluidos com o PVC. Os resultados obtidos mostraram que a morfologia do PVC não foi afetada pelo envelhecimento, também indicam que e possível a difusão dos fluidos dentro da matriz polimérica.

\section{Palavras-chave}

PVC; termogravimetria; calorimetria diferencial de varredura; análise FTIR; RX; envelhecimento. 


\section{Abstract}

Farfán Del Carpio, D. C.; Moraes d'Almeida J. R. (Advisor). Physicochemical degradation of PVC caused by oil derivatives. Rio de Janeiro, 2009. 112p. Dissertação de Mestrado - Departamento Engenharia de Materiais e de Processos Químicos e Metalúrgicos, Pontifícia Universidade Católica do Rio de Janeiro.

The PVC is the unique polymer of great consume that is not $100 \%$ derived from oil, because it contains, by weight, $57 \%$ chlorine (derived from sodium chloride - table salt) and only the remaining $43 \%$ of ethylene is derived from oil. As the matrix rich in chloride is preferred for applications in media environments rich in bacteria, such as pipelines buried or immersed in sea water, the PVC is being used in various applications in the transport of fluids, especially water, but can be also considered an alternative for the transport of other fluids. Thus this work studies the physical - chemical effect aging of poly (vinyl chloride) - PVC, using powder samples exposed to various fluids (water, ethanol and diesel) for various periods of time. The first part of the work was to characterize the material as received and the second the characterization of the material after exposure to fluids. The characterization techniques used were thermogravimetry (TGA), differential thermogravimetry (DTGA), differential scanning calorimetry (DSC), spectroscopic methods (FTIR-ATR) and X-ray diffraction, to measure the physico-chemical interaction of different fluids with PVC. The results showed that the morphology of PVC was not affected by aging, and it can also indicate the diffusion possibility of fluid within the polymer matrix.

\section{Keywords}

PVC; thermogravimetry; differential scanning calorimetry; FTIR analysis; XRD; aging. 


\section{Sumário}

1 Introdução 16

1.1. Objetivo 24

2 Revisão Bibliográfica 25

2.1. Mecanismos de degradação 25

2.1.1. Reações de degradação 26

2.1.1.1. Cisão de cadeias $\quad 26$

2.1.1.2. Degradação sem cisão de cadeias 28

2.1.1.3. Auto-oxidação 30

2.1.1.4. Despolimerização 31

2.1.2. Iniciação das reações de degradação 32

2.1.2.1. Mecânica e termo-mecânica 32

2.1.2.2. Química, foto-química, termoquímica 32

2.1.2.3. Stress-cracking 33

2.1.3. Ensaios e métodos dos processos de degradação 34

2.1.3.1. Métodos de acompanhamento por processos térmicos 35

2.1.3.2. Métodos espectroscópicos 39

2.1.3.2.1 FTIR-ATR 40

3 Caracterização físico - química do PVC 42

3.1. Material 42

3.2. Caracterização do material 43

3.2.1. Análises químicas por espectroscopia FTIR 43

3.2.1.1. Técnica 44

3.2.1.2. Resultados experimentais $\quad 45$

3.2.2. Raios $X$

3.2.2.1. Técnica 49

3.2.2.2. Resultados experimentais $\quad 49$

3.2.3. Análise termogravimétrica (TGA).

3.2.3.1. Técnica 54

3.2.3.2. Resultados experimentais $\quad 55$

3.2.4. Microscopia eletrônica de varredura (MEV) 64

3.2.4.1. Técnica 64 
3.2.4.2. Resultados experimentais

3.2.5. Calorimetria Diferencial de Varredura (DSC) 65

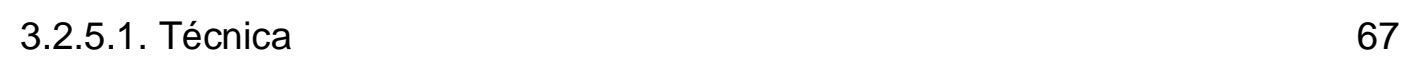

3.2.5.2. Resultados Experimentais 68

$\begin{array}{ll}\text { 3.3. Conclusão } & 70\end{array}$

$\begin{array}{ll}4 \text { Envelhecimento } & 71\end{array}$

4.1. Mecanismos de envelhecimento de um polímero 71

4.1.1. Envelhecimento Químico 71

4.1.2. Envelhecimento Físico 72

4.1.3. Metodologia utilizada para o envelhecimento do PVC 75

4.2. Análise de FTIR do PVC envelhecido 76

4.2.1. FTIR PVC - Água 76

4.2.1.1. FTIR PVC - Água $10-20-45$ dias 76

4.2.2. FTIR PVC - Etanol 79

4.2.2.1. FTIR PVC - Etanol $10-20-45$ dias 79

4.2.3. FTIR PVC - Diesel 82

4.2.3.1. FTIR PVC - Diesel 10 - 20 dias 82

4.3. Difração de Raios X (DRX) do PVC envelhecido 83

4.3.1. Difração de Raios X (DRX) PVC- Água 10 - 20- 45 dias 84

4.4. Análise TGA do PVC envelhecido 84

4.4.1. Caracterização TGA do PVC envelhecido em Água 10 - 20 - 45 dias 85

4.4.2. Caracterização TGA do PVC envelhecido em Etanol 10 - 20 dias 87

4.4.3. Caracterização TGA do PVC envelhecido em Diesel $10-20$ dias 88

4.4.4. Comparação TGA do PVC envelhecido 10 dias em Água, 10 dias em Etanol, e 10 dias em Diesel. 90

4.4.5. Comparação TGA do PVC envelhecido 20 dias em Água, 20 dias em Etanol, e 20 dias em Diesel $\quad 91$

4.5. Análise DSC 93

4.5.1. Análise DSC para o PVC envelhecido em água 93

4.5.2. Análise DSC para o PVC envelhecido em etanol 94

4.5.3. Analise DSC para o PVC envelhecido em diesel 94

4.6. Conclusão 96

5 Conclusão Geral

6 Recomendações para futuros trabalhos 98 
7 Bibliografia

8 Anexos

Anexo I

Anexo 2

Anexo 3

109

Anexo 4

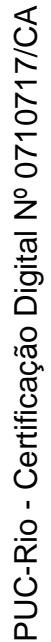




\section{Lista de figuras}

Figura 1 - Rota de obtenção do DCE por cloração direta 16

$\begin{array}{ll}\text { Figura } 2 \text { - Obtenção do MVC a partir do EDC } & 17\end{array}$

$\begin{array}{ll}\text { Figura } 3 \text { - Estrutura química do PVC } & 17\end{array}$

$\begin{array}{lr}\text { Figura } 4 \text { - Fluxograma de fabricação do PVC } & 18\end{array}$

Figura 5 - Embalagens de PVC 20

Figura 6 - Calçados manufaturados de PVC 21

Figura 7 - O PVC nas aplicações médico - hospitalares 21

Figura 8 - O PVC no automobilismo 22

Figura 9 - Diversas aplicações do PVC 22

Figura 10 - Formação do CPVC a partir do PVC 23

Figura 11 - O PVC na construção civil $\quad 24$

Figura 12 - Cisão de ligação C-C: homolítica ou heterolítica. 27

Figura 13 - Mecanismo de degradação sem o rompimento de ligação C-C na $\begin{array}{ll}\text { cadeia principal. } & 28\end{array}$

Figura 14 - Mecanismo de degradação sem rompimento de cadeia para o $\begin{array}{ll}\text { poli(cloreto de vinila). } & 28\end{array}$

Figura 15 - Tipos de defeitos mais comumente encontrados em PVC, dependendo do processo de polimerização. 29

Figura 16 - Processo auto-catalítico na degradação do poli(cloreto de vinila). 30 Figura 17 - Reação de oxigênio com macroradicais alquila, formando radicais peroxila na extremidade ou no meio na cadeia polimérica. 31

Figura 18 - Reação do macroradical peroxila com uma cadeia polimérica 31

Figura 19 - Esquema seqüencial do processo de stress-cracking: 34

Figura 20 - Curvas de TGA para: poli(cloreto de vinila), poli(metacrilato de metila), polietileno e poli(tetrafluoretileno). 37

Figura 21 - Estereoconfiguração da molécula de PVC. 44

Figura 22 - FTIR-ATR Espectro do polímero como recebido 48

Figura 23 - RX Difratograma do PVC como recebido 51

Figura 24 - Formação de polieno a partir da degradação do PVC 52

Figura 25 - Mudanças de massa detectáveis em termogravimetria 55

Figura 26 - Curvas de TG e DTG da degradação térmica do PVC atmosfera de nitrogênio para uma taxa de aquecimento de $5 \stackrel{\circ}{\mathrm{C}} / \mathrm{min} \quad 58$

Figura 27 - Curvas Termogravimétricas típicas do PVC como recebido 59 
Figura 28 - Curvas DTG do PVC como recebido, para 5 diferentes taxas de aquecimento

Figura 29 - Determinação da energia de ativação do PVC para o primeiro pico da curva DTG

Figura 30 - Determinação da energia de ativação do PVC para o segundo pico da curva DTG.

Figura 31- Determinação da energia de ativação do PVC para o terceiro pico da curva DTG.

Figura 32 - Micrografia de uma partícula de PVC como recebido, visualizada no MEV.

Figura 33 - Diagrama esquemático do compartimento da amostra na analise. 66

Figura 34 - Termograma do DSC do PVC como recebido

Figura 35 - Representação esquemática de difusão de um líquido dentro de um polímero

Figura 36 - PVC como recebido a) Tamanho real b) No microscópio com aumento de 35x.

Figura 37 - a) Alimentação do misturador com o PVC; b) Misturador utilizado para o envelhecimento do PVC.

Figura 38 - Espectro IR do PVC envelhecido em água por 10 dias 77

Figura 39 - Espectro IR do PVC envelhecido em água por 20 dias 77

Figura 40 - Espectro IR do PVC envelhecido em água por 45 dias 78

Figura 41 - Comparação dos espectros IR do PVC envelhecido em água durante 10, 20 dias com o PVC como recebido.

78

Figura 42 - Espectro IR do PVC envelhecido em etanol por 10 dias 79

Figura 43 - Espectro IR do PVC envelhecido em etanol por 20 dias 80

Figura 44 - Espectro IR do PVC envelhecido em etanol por 45 dias 80

Figura 45 - Comparação dos espectros IR do PVC envelhecido em etanol durante 10, 20 e 45 dias com o PVC como recebido. 81

Figura 46 - Espectro IR do PVC envelhecido em diesel por 10 dias 82

Figura 47 - Espectro IR do PVC envelhecido em diesel por 20 dias 82

Figura 48 - Comparação dos espectros IR do PVC envelhecido em diesel durante 10 e 20 dias com o PVC como recebido.

Figura 49 - Difratogramas do PVC: como recebido, 20 dias de envelhecido em água, 45 dias envelhecido em água.

Figura 50 - Curvas TGA do PVC envelhecido em água (10, 20 e 45 dias) comparado com o PVC como recebido. 
Figura 51 - Curvas DTG do PVC envelhecido em água (10, 20 e 45 dias) comparado com o PVC como recebido.

Figura 52 - Curvas TGA do PVC envelhecido em etanol (10, 20 dias) comparado com o PVC como recebido.

Figura 53 - Curvas DTG do PVC envelhecido em etanol (10, 20 dias) comparado com o PVC como recebido.

Figura 54 - Curvas TGA do PVC envelhecido em diesel (10, 20 dias) comparado com o PVC como recebido

Figura 55 - Curvas DTG do PVC envelhecido em diesel (10, 20 dias) comparado com o PVC como recebido

Figura 56 - Curvas termogravimétricas comparativas do PVC como recebido e envelhecido em água, etanol e diesel durante 10 dias.

Figura 57 - Curvas DTG comparativas do PVC como recebido e envelhecido em água, etanol e diesel durante 10 dias.

Figura 58 - Curvas termogravimétricas comparativas do PVC como recebido e envelhecido em água, etanol e diesel durante 20 dias Figura 59 - Curvas DTG comparativas do PVC como recebido e envelhecido em água, etanol e diesel durante 10 dias

Figura 60 -Termogramas DSC do PVC como recebido e do PVC envelhecido em água durante 10, 20 e 45 dias.

Figura 61 - Termogramas DSC do PVC como recebido e do PVC envelhecido em etanol durante 10 e 20 dias.

Figura 62 - Termogramas DSC do PVC como recebido e do PVC envelhecido em diesel durante 10 e 20 dias. 


\section{Lista de tabelas}

Tabela 1 Propriedades do PVC

Tabela 2 Alguns exemplos de energias de ligações químicas.

( ${ }^{*}$ para C primário)

26

Tabela 3 Especificações técnicas do NORVIC® SP 1300HP

Tabela 4 Bandas de absorbância mais significativas do PVC comercial no FTIR

Tabela 5 Bandas de absorção para diferentes estruturas presentes no PVC como recebido

Tabela 6 Classificação dos modos $\mathrm{CH}$ e $\mathrm{CH}_{2}$ do PVC sindiotático

Tabela 7 Classificação dos modos do esqueleto e do CCl do PVC sindiotático

Tabela 8 Espaços de redes e intensidade do PVC [resultados obtidos com auxilio do TOPASS]

Tabela 9 Resultados obtidos do TGA para os diferentes estágios de degradação do material como recebido em todas as taxas de aquecimento

Tabela 10 Temperatura de decomposição térmica do PVC a varias taxas de aquecimento.

62

Tabela 11 Resultados da energia de ativação em $\mathrm{kJ} \mathrm{mol}^{-1} \mathrm{em}$ comparação com outros trabalhos

Tabela 12 Energia de ativação para o envelhecimento do PVC em Água $\left(\mathrm{kJ} \mathrm{mol}^{-1}\right.$ )

Tabela 13 Energia de ativação do PVC envelhecido em etanol ( $\left.\mathrm{kJ} \mathrm{mol}^{-1}\right)$

Tabela 14 Energia de ativação do PVC envelhecido em diesel ( $\left.\mathrm{kJ} \mathrm{mol}^{-1}\right)$

Tabela 15 Temperatura de transição vítrea $(\mathrm{Tg})$ do PVC envelhecido em os três diferentes fluidos por 10,20 e 45 dias $\left({ }^{\circ} \mathrm{C}\right)$ 


\section{Lista de símbolos}

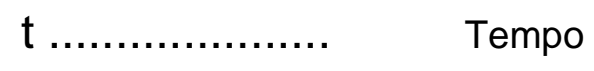

T $\ldots \ldots \ldots \ldots \ldots \ldots . . . . . . . \quad$ Temperatura

Ti ................. Temperatura inicial

$T_{f} \ldots \ldots \ldots \ldots \ldots . . . . . . . \quad$ Temperatura final

$\mathrm{T}_{\mathrm{mi}} \ldots \ldots \ldots \ldots \ldots \ldots . . . . \quad$ Valor do pico de temperatura

Tg ................ Temperatura de transição vítrea

Xsp .................. Coeficiente de interação

V ................... Volume molar do solvente

R .................. Constante dos gases perfeitos

Os ................... Parâmetro de solubilidade do solvente

రp ................... Parâmetro de solubilidade do polímero

Xs .................. Termo de entropia

E $\ldots \ldots \ldots \ldots \ldots \ldots \ldots$ Energia de ativação

$\beta \ldots \ldots \ldots \ldots \ldots . . . . . \quad$ Taxa de aquecimento

A $\ldots \ldots \ldots \ldots \ldots \ldots . . . . . . . \quad$ Fator pré-exponencial

$K$.................. Valor de $\mathrm{K}$ 